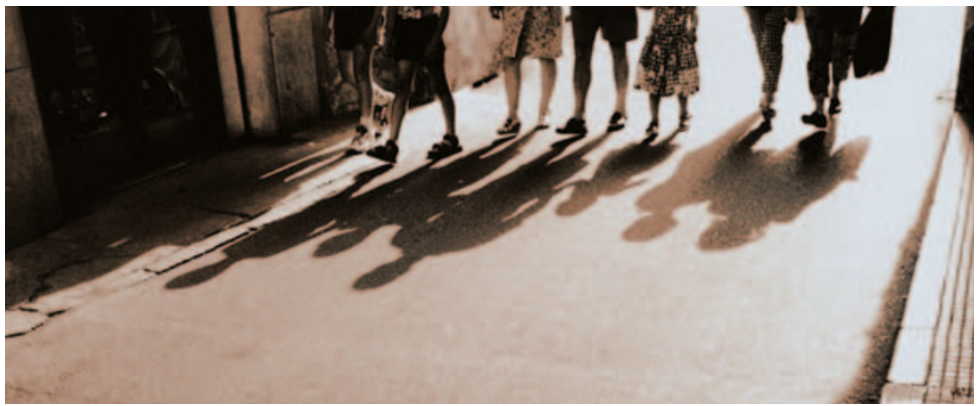

CELL SIGNALLING

\section{Walking the integrin activation road}

Many signalling molecules have been shown to regulate integrin activation, yet the molecular links that connect agonist stimulation with the final steps that lead to integrin activation are not known. Han et al. now describe a pathway from agonist stimulation to integrin activation and establish that Rap1 induces the formation of an 'integrin activation complex' that binds to and activates integrin receptors.

Activation of the platelet integrin $\alpha$ llb $\beta 3$ has been widely used to model activation of the whole integrin family. Platelet agonists stimulate the activation of integrin $\alpha \mathrm{llb} \beta 3$, but they fail to activate recombinant $\alpha$ llb $\beta 3$ expressed in Chinese hamster ovary (CHO) cells or several other nucleated cells.

Given the above discrepancies, Han et al. used a synthetic approach to reconstruct the integrin activation pathway in $\mathrm{CHO}$ cells. Binding of the adaptor protein, talin, is a final step in integrin activation, and platelets express abundant talin compared with most nucleated cells. This prompted the authors to propose that increased levels of talin in $\mathrm{CHO}$ cells enable $\alpha$ llb $\beta 3$ activation in response to a platelet agonist. Analysis of $\alpha$ llb $\beta 3$ expressing $\mathrm{CHO}$ cells that were transfected with talin and/or protein kinase $\mathrm{C} \alpha(\mathrm{PKC} \alpha)$ - which has been implicated as a downstream mediator in the activation of $\alpha \mathrm{llb} \beta 3$ by agonists - showed that increased levels of talin and PKC $\alpha$ promoted agonistinduced talin redistribution as well as activation of $\alpha$ llb $\beta 3$ integrin.

Although talin is a known substrate for PKC, PKC-mediated talin phosphorylation did not affect integrin activation. Instead, the authors found that Rap1 GTPase mediated the activity of PKC in agonist-induced integrin activation. So, is Rap1 the missing link between $\mathrm{PKC}$ and talin in integrin activation? Mutation analysis showed that talin binding to the $\beta$-tails of integrins is required for the activation of $\beta 1$ integrins and that this interaction is required for Rap1-mediated integrin activation. Han and colleagues also showed that active Rap1 GTPase stimulates the membrane localization of talin and enhances the interaction of talin with $\alpha$ llb $\beta 3$ integrin. But, how is Rap1 coupled to talin? Expression of Rap1 effectors in talin-expressing cells revealed that the Rap1 effector RIAM mediates the formation of a Rap1-induced integrin activation complex, which contains talin and RIAM, that binds to and activates the integrin.

The authors proposed that "agonist receptors promote ... the activation and/or translocation of active GTP bound Rap1 to the plasma membrane through activation of PKC or a Rap GEF. At the plasma membrane, activated Rap1 interacts with RIAM, leading to the recruitment of talin to form the integrin activation complex, thus unmasking the integrin binding site in talin leading to integrin activation." This pathway can now be used as a template to integrate different agonists and signalling pathways that control integrin activation.

\section{Ekat Kritikou}

ORIGINAL RESEARCH PAPER Han, J. et al. Reconstructing and deconstructing agonistinduced activation of integrin $\alpha$ llb $\beta 3$. Curr. Biol. 16, 1796-1806

WEB SITE

Mark H. Ginsberg's laboratory:

http://pstp.ucsd.edu/ginsberg/about.html
Crystal structure of a rhomboid family intramembrane protease.

Wang, Y. et al. Nature 11 Oct 2006 (doi:10.1038/nature05255)

The Escherichia coli protein GlpG is a rhomboid-family protease that cleaves the transmembrane domain of membrane-protein substrates. The 2.1-Å crystal structure of the GlpG core domain, which consists of six transmembrane domains, provides new insights into the mechanism of intramembrane proteolysis. The catalytically active site of GlpG is embedded in the membrane, and the substrate is proposed to dock laterally between two transmembrane helices, replacing a loop structure that functions as a gate that blocks the lateral opening of the active site.

\section{$\Rightarrow$ MEMBRANE TRAFFICKING}

Exomer: a coat complex for transport of select membrane proteins from the trans-Golgi network to the plasma membrane in yeast.

Wang, C.-W. et al. J. Cell Biol. 174, 973-983 (2006)

Wang and colleagues uncovered a new type of coated vesicle that transports the yeast plasma-membrane protein Chs 3 directly from the trans-Golgi network (TGN) to the plasma membrane of the mother-bud neck. Chs 5 and Chs6, as well as three Chs6 paralogues, form a complex that is required for the transport of Chs3. Coat assembly is initiated by the activation of a G protein of the Arf family; indeed, Sec7 - a TGN-localized Arf1 nucleotide-exchange factor - is required to recruit Chs 5 to the TGN surface. The coat complex was visualized by electron microscopy and was named exomer for its role in exocytosis from the TGN to the cell surface.

\section{$\Rightarrow$ DNA REPAIR}

Direct observation of individual RecA filaments assembling on single DNA molecules.

\section{Galletto, R. et al. Nature 20 Sep 2006 (doi:10.1038/nature05197)}

Recombinational DNA repair in Escherichia coli involves the formation (or 'nucleation') of a RecA nucleoprotein filament on DNA, followed by elongation. Galletto et al. visualized filament assembly on individual double-stranded DNA molecules using fluorescently modified RecA. Nucleation of RecA filaments is independent of ATP hydrolysis but dependent on the type of nucleoside triphosphate and the RecA concentration. By contrast, individual RecA filaments can grow bidirectionally in the presence of ATP, and this growth is independent of the nucleoside triphosphate.

\section{MECHANISMS OF DISEASE}

Caveolin- 1 is essential for liver regeneration.

Fernăndez, M. A. et al. Science 313, 1628-1632 (2006)

Caveolin-1 is an essential component of caveolae - distinct plasma-membrane domains that are involved in processes such as signalling and membrane sorting. Caveolin-1-knockout mice showed low survival and impaired liver regeneration after partial hepatectomy. This phenotype was associated with an intracellular lipid imbalance, reduction in lipid-droplet accumulation and a failure by cells to progress through the cell cycle. Caveolin-1 is therefore proposed to have an important role in coordinating lipid metabolism with the proliferative response during liver regeneration. 